\title{
Als Arzt in Simbabwe
}

\author{
H. Spycher
}

Im Frühsommer dieses Jahres hatte ich Gelegenheit, einen Entwicklungshilfeeinsatz in einem Spital in Simbabwe zu leisten. Ich verbrachte fast zwei Monate im Muvonde Hospital in Driefontein. Anbei meine Erlebnisse.

Nun bin ich bereits vierzehn Tage im Muvonde-Missionsspital in Driefontein tätig und konnte erste Eindrücke und Erfahrungen sammeln.

Simbabwe, die ehemalige englische Kolonie Südrhodesien, ist rund zehnmal so gross wie die Schweiz, hat aber nur 13 Millionen Einwohner. Die offizielle Amtsprache ist ein allerdings nur schwerverständliches Englisch. Ich habe immer noch Verständigungsschwierigkeiten, da viele Leute auf dem Land dieser Sprache nicht mächtig sind, so dass ich im Spital auf die Übersetzung durch Krankenschwestern und Pfleger angewiesen bin.

Das Leben in Simbabwe ist sehr teuer geworden. Seit die weissen Farmer vertrieben worden sind, die $60 \%$ des Volkseinkommens erarbeitet haben, liegt die Wirtschaft danieder. Seit einem Jahr beträgt die Inflationsrate 600\%. Ein Arbeiter verdient etwa 100000 Dollar im Monat, entsprechend 25 Franken, die nicht mehr zum Leben reichen. So kostet ein Liter Milch 4000 Dollar und ein Pfund Tomaten 7800 Dollar. 80\% der männlichen Bevölkerung sind allerdings arbeitslos.

Die meisten Spitäler sind staatliche Einrichtungen. Die ärmere Bevölkerung kann sich die Behandlung in diesen Spitälern aber nicht leisten, schon gar nicht eine Krankenversicherung.

Patienten werden dort erst behandelt, wenn sie eine Zahlung von 2 Millionen Dollar geleistet haben. Eine Ultraschalluntersuchung zum Beispiel kostet 400000 Dollar (vierfacher Monatslohn eines Arbeiters). Den meisten Patienten fehlt das Geld, und so sind sie auf die Behandlung in Missionsspitälern angewiesen, in denen die Behandlung sehr billig oder sogar gratis ist. Es gibt etwa 100 Missionsspitäler in Simbabwe. So kommen eben viele Patienten aus weiter Entfernung zu uns ins Spital, um sich behandeln zu lassen. Mit allerlei Transportmitteln, z. B. mit dem Bus, der allerdings teuer ist, mit Eselskarren oder viele auch zu Fuss, versuchen sie das Spital zu erreichen. Das Transportfahrzeug des Spitals, das für allerlei Transporte diente (Patienten von der $18 \mathrm{~km}$ entfernten Busstation abholen,
Waisenkinder einsammeln, blinde Patienten zur Augenoperation bringen usw.), wurde gestohlen und der Fahrer dabei niedergeschlagen und schwer verletzt. Nun fehlt das Geld für ein neues Fahrzeug. Es fehlt überhaupt alles. Da wir von der Regierung nur 1800 Franken monatlich für den Betrieb des 180-Betten-Spitals erhalten, sind keine grossen Sprünge möglich. Ohne Spenden von Firmen oder Privatpersonen könnten wir nicht überleben. So fehlt es an Verbandmaterial, diagnostischen Instrumenten und vor allem an Medikamenten. Viele Krankheiten können nicht behandelt werden, weil die Medikamente dafür nicht vorhanden sind.

Das Muvonde-Spital befindet sich auf $1500 \mathrm{~m}$ über dem Meer. Man nennt das Gebiet Hochsavanne. Wegen der hohen Lage gibt es hier glücklicherweise keine Malaria. Da es Winter ist, sind die Nächte aber bitterkalt; eine Heizung gibt es in den Häusern und im Spital nicht. Die Patienten verkriechen sich deswegen unter die Wolldecken.

Arztpraxen gibt es keine. Es gibt aber Aussenstationen des Spitals, Clinics genannt. Das sind einfache Gebäude, wo Krankenschwestern Patienten betreuen, da die Distanzen einfach zu gross sind, um für die täglichen Gebresten das Spital aufzusuchen. Wir sehen bei uns also nur Patienten mit ernsthaften Krankheiten oder Unfallfolgen.

Dr. Aschwanden, ehemals Arzt in Stein am Rhein, ist medizinischer Leiter des Spitals. Er ist ein gut ausgebildeter Chirurg, der von der Augenoperation bis zur Prostataoperation alle gängigen Operationen beherrscht. In der Schweiz sind solche Operationen den spezialisierten Chirurgen vorbehalten, aber die gibt es hier eben nicht. Zwei weitere Ärzte, ein Nigerianer und ein Simbabwer, arbeiten am Spital. Es ist aber schwierig, Ärzte zu finden, da alle Akademiker auszuwandern versuchen.

Der Arbeitstag beginnt im Operationssaal. Um 8.15 Uhr wäre Operationsbeginn. Ich bin an schweizerische Pünktlichkeit gewöhnt und somit immer der erste Anwesende. Wir sind hier aber in Afrika, und so ist Warten angesagt. Es gibt hier ein Sprichwort: «Den Weissen gab Gott die Uhr, den Afrikanern die Zeit.»

Die erste Operation, eine beidseitige Hydrozele, ist für mich bestimmt. Ich muss also beweisen, was ich letzte Woche gelernt habe. Es 
geht ganz gut, wenn man die Anatomie und den Operationsgang einmal begriffen hat.

Anschliessend folgt eine Hysterektomie. Die ist dem Chef vorbehalten, da der riesige Uterus myomatosus den ganzen Bauch ausfüllt und man Schwierigkeiten hat, die A. uterinae unterbinden zu können. Es gibt einigen Nervenkitzel, aber die Operation gelingt. Zwei Tage später sieht man die Patientin schon munter herumlaufen. Die Afrikaner sind eben wenig schmerzempfindlich.

Dann bittet mich Dr. Aschwanden, eine Ultraschalluntersuchung zu machen. Eine 25jährige Frau ist mit einem riesigen Bauch notfallmässig eingeliefert worden. Klinisch handelt es sich um Ascites.

Mit dem Ultraschall ist es so eine Sache. Wir haben ein ungefähr 15 Jahre altes Gerät, zudem einen nicht geeigneten Schallkopf. Es ist also auch für mich als erfahrenen Ultraschallanwender schwierig, richtige Diagnosen zu stellen. Häufig sieht man ausser Schneegestöber gar nichts. Wir hoffen immer noch, dass uns ein Spital oder eine Firma in der Schweiz ein Occasionsgerät gratis zur Verfügung stellt.

Die Diagnose Ascites bestätigt sich. Aber was ist die Ursache? In Europa würde man an einen bösartigen Tumor denken; für Alkoholmissbrauch ist die Patientin zu jung.

Laboruntersuchungen oder ein Computertomograph stehen nicht zur Verfügung. Es bleibt uns also nichts anderes übrig, als eine Probelaparotomie zu machen. Ein grässlicher Anblick: Sämtliche Därme und die übrigen Bauchorgane sind übersät mit kleinen Knötchen. Es handelt sich um eine sogenannte miliare Tuberkulose (Milium $=$ Hirsekorn). Ein Krankheitsbild, das wir bei uns wohl nie mehr sehen.

Nun ist doch noch Zeit zum Mittagessen. Wir nehmen dieses stehend im Sterilisierraum des Spitals ein. Es gibt Sadza (Polenta) und Kohl. Man isst mit den Händen. Mais ist hier das Hauptnahrungsmittel. Zum Frühstück gibt es bei den Afrikanern Maisporridge, am Mittag Sadza und zum Abendessen auch Sadza, und das jeden Tag.

Am Nachmittag ist Poliklinik. In Reihen warten die Patienten geduldig, bis sie drankommen. Der erste Patient ist ein 8jähriger Junge. Er ist im Gang zusammengebrochen und wird im Rollstuhl hereingebracht. Bei uns wäre er weiss wie ein Leintuch. Bei den Afrikanern sieht man wegen der Hautfarbe die Blutarmut nur an den Augen, an der Zunge und an den Fingernägeln. Der Junge hat nur noch ein Hb von 4,4 g/dl. Auch hier müssen wir vorerst raten, da uns zur Diagnose die notwendigen Laboruntersuchungen fehlen. In Frage kommen eine Malaria, eine
HIV-Infektion, Fehlernährung, aber auch eine chronische Infektion. Um das Schlimmste abzuwenden, erhält er unsere letzte Flasche Blut. Alle Patienten, die eine Bluttransfusion benötigen, erhalten Blut mit der Blutgruppe 0, sofern wir überhaupt Blut zur Verfügung haben.

So geht es den ganzen Nachmittag weiter. Am Abend ist man erschöpft und froh, ins Bett zu kommen; notabene das einzige warme Plätzchen im Haus.

Mai bis Juli sind hier die Wintermonate, und so ist es am folgenden Tag sehr kalt, ein richtiger «Novembertag» eben: Der Himmel ist grau verhangen, man friert ständig.

Zuerst machen wir Visite im Regionalhaus der Immensee-Mission. Dort leben viele Patres und Fratres, die hier ihren Lebensabend verbringen. Sie sind dankbar für einen kurzen Besuch und einige nette Worte. Sie leben von der AHV, sind aber immer wieder grosszügig und spenden Geld fürs Muvonde-Spital.

\section{Augenkrankheiten in den Entwicklungs- ländern}

Wenn wir über Krankheiten in den Entwicklungsländern sprechen, so denken wir immer an Durchfall, Malaria und andere gefährliche Infektionskrankheiten, woran alljährlich mehrere Millionen Patienten, vor allem Kinder, sterben. Eine Krankheitsgruppe wird aber gerne vergessen: die Augenkrankheiten. Augenleiden und nachfolgende Blindheit sind hier weit verbreitet. Auch in der reichen Schweiz ist ein Blinder durch sein Schicksal schwer getroffen. Wir haben bei uns aber Möglichkeiten zur Verfügung, ihr Leben zu erleichtern: Blindenschulen, Blindenhunde, Invalidenversicherung usw. Das fehlt hier alles. Der Blinde ist auf seine Familie angewiesen, sofern er noch eine hat. Es sterben ja zurzeit in Simbabwe ungefähr 3000 Aidskranke pro Woche.

Man rechnet, dass es 30 Millionen Blinde auf der Welt gibt; davon leben 29 Millionen in den Entwicklungsländern.

Die Ursachen der Blindheit sind: grauer und grüner Star, Infektionskrankheiten und Mangelernährung.

Durch geeignete Vorbeugungs- und Therapiemassnahmen liessen sich aber viele Augenkrankheiten in den Entwicklungsländern verhindern.

Die Augenkrankheiten sind das «Hobby» unseres Chefs, Dr. Aschwanden. Er hat im Spital von Driefontein einen guten Augenservice aufgezogen. Am Freitag wird jeweils nicht operiert, und so fahre ich mit der "Augenschwester» 


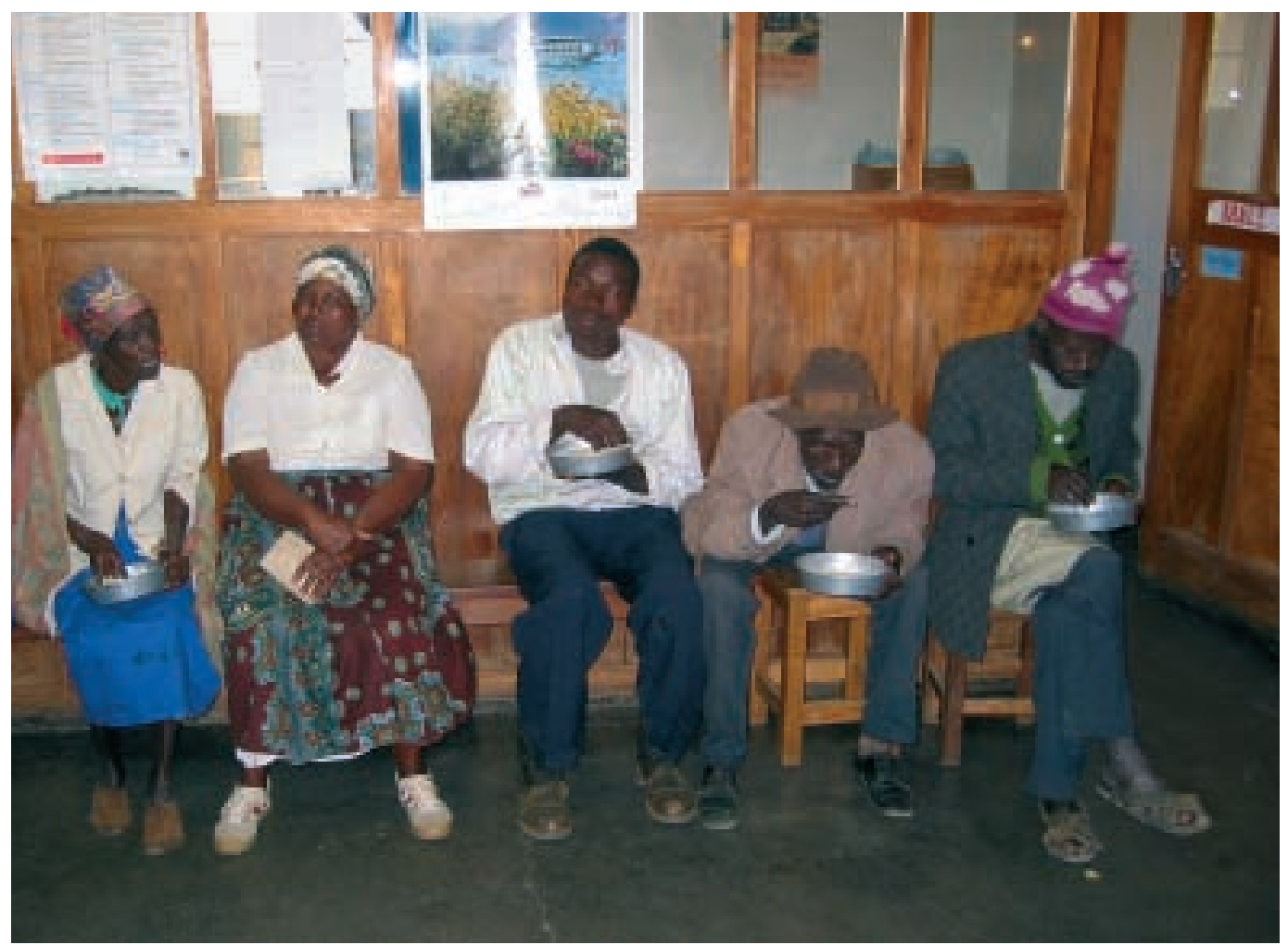

Poliklinikpatienten beim Essen.

Rubaya (sie ist auch gleichzeitig noch Narkoseschwester und Hebamme) über Land in eine $100 \mathrm{~km}$ entfernte Aussenstation: eine kleine Hütte, wo eine Schwester, vergleichbar mit unseren Gemeindeschwestern, jeweils «Sprechstunde» macht. Dort warten nun etwa 140 Patienten auf uns. Was man da als Arzt zu sehen bekommt, ist schrecklich. Total eingetrübte Augen, Entzündungen jeder Art und Augentumoren. Die zu operierenden Patienten werden gleichentags im Kombiwagen, eingepfercht wie Sardinen, zur Operation mitgenommen. Bei den anderen geht es ums Verschreiben einer Brille. Die meisten sind alterssichtig und können nicht mehr lesen. Im Schnellzugtempo werden Brillen angepasst und gleich abgegeben. Eine Brille würde beim Optiker in Harare (Hauptstadt von Simbabwe) zwischen 500000 und einer Million SimbabweDollar kosten (wir erinnern uns: Der Monatslohn eines einfachen Arbeiters beträgt ungefähr 100000 Dollar). Wir sind in der Lage, die Brillen für 10000 Dollar, also etwa Fr. 2.50, abzugeben. Dies ist möglich, weil uns die Firma Knecht in Stein am Rhein grosszügigerweise eine Einrichtung zum Brillenschleifen geschenkt hat, Optiker in der Schweiz uns alte Brillengestelle zur Verfügung gestellt haben und wir die Gläser für eine Brille in Indien für Fr. 1.50 einkaufen können. Diese werden dann anschliessend in unserer
Schleiferei in die Brillengestelle eingepasst. Ohne Hilfe aus der Schweiz könnten wir diesen Patienten nicht helfen.

Es ist bereits dunkel, als wir über die löchrige Sandpiste wieder zurückfahren. Es gibt praktisch keine Wegweiser, und ich staune, wie der Chauffeur, Herr Makuve, unser Mann für alles, seinen Weg im Dunkeln findet.

Am darauffolgenden Montag ist jeweils Augenoperationstag. Dr. Aschwanden zeigt mir die Operation eines grauen und eines grünen Stars. Er macht das hervorragend und mit wenigen technischen Einrichtungen. Am Schluss ist noch ein «totes» Auge zu entfernen, eine Operation, die für mich bestimmt ist.

Es wird einem also hier in Afrika nie langweilig, und man lernt eine Menge. Ich führe hier Operationen durch, auf die in der Schweiz ein Assistent Jahre warten müsste. Bereits am zweiten Aufenthaltstag musste ich eine Sectio machen, und nach zwei Wochen kam bereits die erste Dünndarmresektion und Anastomose auf mich zu.

\section{Eine Schwesternschule im Busch}

In der folgenden Woche kam viel Arbeit auf mich zu. Einer der Ärzte ging ins verlängerte Wochenende, der andere kam nicht aus den Ferien zu- 


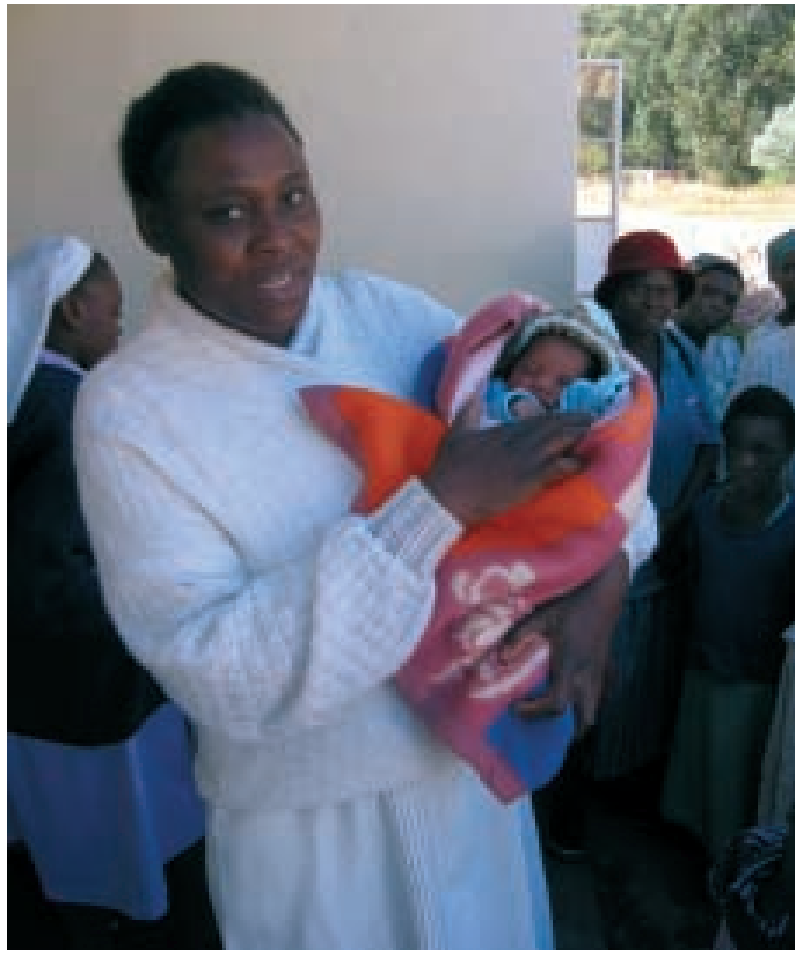

«Augenschwester» Rubaya.

rück. Es ist halt alles afrikanisch hier, mit der Zeit nimmt man es nicht so genau. So war ich also «Herr» über etwa hundert Spitalpatienten. Das hiess Visite auf der Männer-, der Frauen- und Wöchnerinnen- und der Kinderabteilung machen, dazu kamen etwa 30 ambulante Patienten, ein Drittel davon schwere Tuberkulose- und Aidspatienten. Zwischendurch in den Operationssaal, um zwei riesige Abszesse zu eröffnen. Ich habe in meinem Leben noch nie so grosse Abszesse gesehen wie hier in Afrika. Man kann sich kaum vorstellen, was für Schmerzen die Kranken aushalten mussten, bis sie endlich ins Spital gelangten. Am Ende des Tages noch einmal ein Rundgang über die Abteilungen, um nach dem Rechten zu sehen.

Ich arbeite sehr gerne mit den schwarzen Schwestern und Pflegern zusammen. Sie sind sehr nett und hilfsbereit, wenn man ihre Mentalität versteht und sich an die Gegebenheiten anpasst. Wir haben allerdings mit einem grossen Personalproblem zu kämpfen: Für das 180Betten-Spital stehen nur fünf ausgebildete Schwestern zur Verfügung. Die übrigen sind angelerntes Hilfspersonal.

Für die etwa 3500 Spitaleintritte pro Jahr bräuchten wir etwa 50 ausgebildete Schwestern. Leider wandern diese ebenfalls in andere Länder, vor allem nach Südafrika und Europa, aus, da sie dort die besseren Arbeits- und Lebensbedingungen haben.
Dank einer grossen Spende eines deutschen Ehepaares kann nun Dr. Aschwanden eine Schwesternschule bauen, deren Betrieb von der Regierung unterstützt werden wird. Das ermöglicht in Zukunft die Ausbildung von 90 Schwestern und Pflegern in einem dreijährigen Ausbildungslehrgang. Diese müssen sich dann verpflichten, drei Jahre in einem Missionsspital zu arbeiten. Mit einer solchen Ausbildungsstätte können in Zukunft Schwestern und Pfleger an viele Missionsspitäler in Simbabwe vermittelt werden.

Der Gebäudekomplex umfasst fünf Schlafhäuser (Dormitories), einen Küchen-/Esssaalbereich, ein Schulgebäude, ein Haus für die Unterkunft der Lehrer sowie für die Verwaltung.

Die Kosten für den Bau können relativ tief gehalten werden, da das Land kostenlos abgegeben wurde und Bruder Serafin von der ImmenseeMission die Architekturarbeiten gratis ausgeführt hat. Man rechnet mit Baukosten von 1,3 Millionen Franken; in der Schweiz müssten wir wohl mit dem Zehnfachen rechnen.

Natürlich haben wir auf dem Bau mit einigen Schwierigkeiten zu kämpfen. Es gibt keine Baumaschinen, vieles ist Handarbeit. Alles geht langsamer vor sich. Zudem musste das Baugelände mit einem hohen, stacheldrahtbewehrten Zaun umgeben werden und wird nachts und an Wochenenden bewacht, sonst würde alles gestohlen, was nicht niet- und nagelfest ist.

Wegen der hohen Schwindsucht des Geldes (Simbabwe hat die höchste Inflationsrate der Welt) sind die Preise für die Löhne und das Baumaterial massiv gestiegen. Der Preis für einen Sack Zement zum Beispiel ist innert weniger Monate von 4000 auf 45000 Simbabwe-Dollar gestiegen. Kein Unternehmer gibt mehr verbindliche Offerten $a b$, da dies sein Ruin sein würde.

Das bedeutet natürlich, dass wir für den Bau mit einer erheblichen Kostenüberschreitung rechnen müssen. Trotzdem sollte die Schule in etwa einem Jahr eingeweiht werden können.

Im Moment fehlen nur noch die finanziellen Mittel für die Inneneinrichtungen (Stühle, Bänke, Betten usw.). Wir hoffen natürlich, dass der Fehlbetrag (etwa 300000 Franken) noch durch Spenden aufgebracht werden kann.

\section{Ein Selbstmord wegen 90 Rappen}

Hier in Afrika erlebt man jeden Tag Spannendes, Trauriges, aber auch Schönes, gelegentlich sogar Wunder. So wurde uns vor zwei Tagen aus dem $60 \mathrm{~km}$ entfernten St.-Theresa-Spital ein hübsches 18jähriges Mädchen überwiesen. Bei ihrem Ein- 
treffen war sie tief bewusstlos. Beim Befragen der Eltern stellte sich heraus, dass die Familie kein Geld hatte, um der Tochter eine Schulprüfung zu finanzieren. Die Prüfung hätte 3700 Dollar (etwa 90 Rappen) gekostet. Hier in Simbabwe muss man nämlich in einer öffentlichen Schule für alles und jedes bezahlen. Aus Verzweiflung wollte sich das Mädchen deswegen mit einem Insektenvertilgungsmittel, einer organischen Phosphatverbindung, das Leben nehmen. Diese Insektizide gelten als äusserst giftig und wirken bereits in kleinen Dosen tödlich. Wir haben ihr Atropin in hohen Dosen infundiert. Es half alles nichts. Im Laufe von 24 Stunden verschlechterte sich der Zustand des Mädchens, und sie wies bereits Cheyne-Stokes-Atmung auf. Wir hatten sie aufgegeben und den geplagten Eltern die Schwere des Krankheitsbildes dargelegt und gesagt, dass kaum Hoffnung bestünde.

Zwei Tage später wollte ich gerade mit der Visite beginnen, als mich eine Krankenschwester wegen des Mädchens im Gang aufhielt. Sie sagte zu mir: «Doctor, the girl is hungry, may she eat?» Ich fiel aus Wolken, als ich das totgeglaubte, allerdings noch stark verwirrte Mädchen aufrecht im Bett sitzen sah. Eine Sternstunde für mich als Arzt. Natürlich habe ich den Eltern das Geld für die Prüfung und für kommende weitere Prüfungen gegeben. Man kann sich die Freude des Mädchens und dessen Eltern kaum vorstellen. Der Betrag, für mich einige Franken, für simbabwische Verhältnisse aber viel Geld.

Leider erlebt man nicht jeden Tag solche Wunder, obwohl ich erstaunt bin, wie selbst schwere Krankheitsbilder bei den Afrikanern durch einen kleinen ärztlichen «Schupf» $\mathrm{ab}$ heilen können.

Der Alltag ist geprägt durch die Aidskranken. Man schätzt, dass von den 13 Millionen Menschen in Simbabwe rund 3 Millionen mit dem HI-Virus angesteckt sind. Wahrscheinlich ist diese Zahl sogar zu tief gegriffen. So leiden etwa $60 \%$ meiner Spitalpatienten unter den Folgeerscheinungen von Aids, vor allem Tuberkulose, Kryptokokken-Meningitiden und Kaposi-Sarkomen.

Die HIV-Infektion können wir leider nicht behandeln, da das Geld für die sehr teuren Medikamente einfach fehlt. Glücklicherweise hat uns eine holländische Wohltätigkeitsorganisation die Medikamente gegen die Tuberkulose zur Verfügung gestellt, so dass wir hier wenigstens die Tuberkulose behandeln können. Ich könnte bald ein Lehrbuch über alle seltenen Formen von Tuberkulose verfassen.

Leider hat die Aidskrankheit noch eine andere Folgeerscheinung, über deren Ausmass man sich kaum ein Bild machen kann. Da die meist jungen Eltern einfach hinwegsterben, bleiben die Waisenkinder übrig, sofern sie nicht ebenfalls an Aids gestorben sind. Unser Spital betreut rund 1000 Aidswaisenkinder aus der näheren und weiteren Umgebung. Wenn noch Grosseltern vorhanden sind, können die Kinder bei ihnen leben. Den Grosseltern fehlt aber meistens das Geld für die Ernährung der Kinder, da sie keine Arbeit und somit kein Einkommen haben. Eine AHV gibt es nicht.

Hier springt nun unser Spital in die Bresche. So fragte mich Schwester Rubaya, ob ich zu den Waisenkindern mitkommen wolle; es würden etwa 200 Kinder im Sanatorium erwartet.

Natürlich ging ich mit. Von überall her kamen die Kinder jeglichen Alters. Sie hatten Distanzen bis zu $20 \mathrm{~km}$ zu Fuss zurückzulegen. Ein trostloses Bild! Kleine Kinder in zerlumpten Kleidern trugen teilweise noch kleinere Kinder auf dem Rücken.

Den ganzen Nachmittag haben wir ihnen Hosen und Hemden, die wir aus Deutschland gespendet bekommen hatten, abgeben. Es passte natürlich nicht alles, aber die Kinder strahlten vor Dankbarkeit. Am Schluss kriegten noch alle zu essen, natürlich Sadza. Leider reicht die Maisernährung nicht aus, um die Bedürfnisse der Kinder zu decken. Sie bräuchten auch Milch, da sonst eine Eiweissmangelkrankheit (Kwaschiorkor) entsteht. Man sieht den Kindern diese Krankheit sofort an: Sie haben riesige Wasserbäuche und dünne Beinchen. Jede Woche habe ich auf der Kinderabteilung solche Kinder zu betreuen.

Ich habe in Simbabwe viel gelernt. Ich musste Medizin mit bescheidenen Mitteln betreiben. Ich habe auch erfahren, wie gross das Elend in der dritten Welt ist und wie gut es uns in der Schweiz geht. Mit wenig Geld könnten wir vielen Simbabwern das Überleben sichern. Es ist damit zu rechnen, dass in den nächsten Monaten wieder eine Hungersnot ausbricht und viele Menschen, vor allem Kinder, daran sterben werden.

\section{Tun wir etwas dagegen!}

Wir haben einen Verein gegründet, dessen Mitarbeiter alle ehrenamtlich arbeiten. Jeder gespendete Franken kommt ohne administrative Abzüge dem Muvonde-Spital und dem Lebenswerk von Dr. Aschwanden zugute. Spendenkonto: «Hilfe für Simbabwe», Stein am Rhein, PC 82-8018-6. 